\title{
A set of novel decision-making indices to freeze all embryos or to continue fresh embryo transfers upon each condition in the individualized controlled ovarian stimulation cycles
}

\author{
Hisao Ando, Hiroko Minamoto, Noriko Suzuki \\ Center for Reproductive Medicine and Surgery, Toyohashi Municipal Hospital, Toyohashi, \\ JAPAN
}

Problem statement: Freeze-all policy is a novel approach to improve assisted reproductive technology (ART) outcome based on that controlled ovarian stimulation may have a negative effect on the receptivity of the endometrium for embryo implantation. However, overall benefits and limitations of the new strategy have not been elucidated. We have created a set of novel formulae to consider the policy upon each condition at every ART facility. Methods: We have set up two indices: the freezeall suggestion (FAS) and the freeze-all consideration (FAC). Each index is calculated by the formula as shown in Figure 1. We applied the indices in our own practice after the data of conventional freeze-all cycles, such as those for avoiding ovarian hyperstimulation syndrome (OHSS) or those due to the thin endometrium, had been eliminated. Results: Overall FAS and FAC index calculated from our most recent 896 cycles was 18.6 and 59.7, respectively. FAS and FAC index in women 35 years old and over (481 cycles) was 17.2 and 63.8, respectively. FAS and FAC index in women younger than 35 (415 cycles) was 20.2 and 54.9, respectively. FAS and FAC index in women with peak estradiol (E2) concentration $3,500 \mathrm{pg} / \mathrm{ml}$ or more (162 cycles), where we currently freeze all embryos if E2 is increased above $5,000 \mathrm{pg} / \mathrm{ml}$, was 27.7 and 71.6 , respectively. FAS and FAC index in women undergoing $\mathrm{GnRH}$ agonist/antagonist (509/287 cycles) was $22.8 / 13.2$ and $61.5 / 60.3$, respectively.
Conclusion: The new indices helped to compare the merit of freeze-all application upon each condition. In our protocol, the benefit of freeze-all policy may exist under the condition of lower peak E2 than that for avoiding OHSS. GnRH antagonist cycles did not show any advantage of the freeze-all policy in our practicetortor.

FAS index = (2) $\div((1)+(2)+(3)) \times$
FAC index = (2) $+(3)) \div((1)+(2)+(3)) \times$
\begin{tabular}{|c|c|c|}
\hline & ET & $\begin{array}{c}\text { Following } \\
\text { TET(s) }\end{array}$ \\
\hline$(1)$ & Yes & - \\
\hline$(2)$ & No & Yes \\
\hline$(3)$ & No & No \\
\hline
\end{tabular}

Figure 1. Proposed indices to evaluate freeze-all decisions. FAS: freeze-all suggestion. FAS: freeze-all consideration. (1)(2) (3): fresh cycle number. ET: fresh embryo transfer. TET: frozenthawed embryo transfer. Yes/No: clinical pregnancy without ectopic pregnancy.

TABLE 1. FAS and FAC indices and related cycle numbers in various COS cycle conditions.

\begin{tabular}{|l|c|c|c|c|c|c|}
\hline Condition & All cycles & (1) cycles & (2) cycles & (3) cycles & FAS index & FAC index \\
\hline Overall & 896 & 361 & 167 & 368 & 18.6 & 59.7 \\
\hline Female age $\geqq 35$ & 481 & 174 & 83 & 224 & 17.2 & 63.8 \\
\hline Female age $<35$ & 415 & 187 & 84 & 144 & 20.2 & 54.9 \\
\hline Estradiol $\geqq 3,500 \mathrm{pg} / \mathrm{ml}$ & 162 & 46 & 45 & 71 & 27.7 & 71.6 \\
\hline GnRH agonist protocol & 509 & 196 & 116 & 197 & 22.8 & 61.5 \\
\hline GnRH antagonist protocol & 287 & 114 & 38 & 135 & 13.2 & 60.3 \\
\hline
\end{tabular}

